\title{
Reducing fuel consumption by using a new fuel-efficiency support tool
}

\author{
Mascha van der Voort ${ }^{1)}$ and Mark Dougherty ${ }^{2)}$ \\ ${ }^{1)}$ Department of Civil Engineering \& Management \\ University of Twente \\ ${ }^{2)}$ School of Transportation \& Society \\ Högskolan Dalarna \\ P.O. Box 217, 7500 AE Enschede, The Netherlands \\ Tel: +31 534894322 or Fax: + 31534894040 \\ E-mail: m.c.vandervoort@sms.utwente.nl \\ S-78 188 Borlänge, Sweden \\ Tel: +4623778500 \\ E-mail: mdo@du.se
}

\section{Abstract}

A fuel-efficiency support tool has been designed, which includes a normative model describing optimal driver behaviour for minimising fuel consumption. If actual behaviour deviates from optimal behaviour, the system presents advice on how to change behaviour. Evaluation revealed that drivers used $\sim 16 \%$ less fuel compared with 'normal driving'.

\section{Introduction}

Energy consumption in its present volume and composition is using up our scarce resources. Furthermore, the pollution resulting from this energy consumption is a burden on the environment. Although several new energy-supply technologies are emerging, the world's consumption of fuels derived from natural oil continues to increase every year. Oil is, however, one of our finite resources. Therefore, fuel conservation, that is performing the same (or similar) transport task with the consumption of less fuel, is a sensible strategy to reduce environmental stress.

Several methods exist for reducing the fuel consumption of road transport. The largest potential to improve fuel economy probably lies in improving vehicle technology [1]. However, such measures have a long implementation time. The most effective way to reduce fuel economy in the short term is to aim at a change in driver behaviour, which can lead to a reduction in fuel consumption of up to 15\% [2]. An additional benefit of aiming at a change in driver behaviour is that the improvement achieved will still be valid when new vehicle technology becomes available. Together they can then reduce fuel consumption even further.

To induce a more optimal driver behaviour, the driver must be provided with feedback. Feedback cannot only help to obtain the desired behaviour, but also to maintain this behaviour in the long term. A fuel-efficiency support tool is an effective way to provide this feedback.

Several driver support tools have been developed in the past to directly or indirectly improve fuel economy. However, a review of available devices revealed that none of the devices was able to bring about the levels of fuel reduction judged possible, because of some major shortcomings. Van der Voort [3] concluded that for a driver support tool to significantly improve fuel economy, it should:

- provide the driver with clear, accurate and non-contradictory information

- take into account the present context of the vehicle

- place no requirements on the driver which are too high to safely combine with the actual driving task

- work within both an urban and non-urban environment.

One potential way to meet these requirements is to provide the driver with direct information on how to drive more fuel-efficiently. 


\section{A new-generation fuel-efficiency support tool}

In this research project, a new-generation fuel-efficiency support tool has been designed, which takes into account the previously described requirements. The proposed support tool is a purely advisory system. The driver can decide whether to accept the advice given by the support tool.

In this section, the basic architecture of the proposed support tool will be described. The support tool compromises three basic components:

1. inputs

2. data processing module

3. human-machine interface.

Inputs

The inputs to the system can be divided into two categories: measured inputs and system parameters. As far as is possible, any fuel-efficiency support tool should use measured inputs which are readily available from existing in-vehicle systems and technology. Therefore only parameters such as vehicle speed, engine speed, clutch, gear position, accelerator position, steering angle, braking force and headway were used as an input to the system.

Headway is not directly needed to determine fuel consumption. However, headway is a vital measurement if one wishes to determine whether the proposed advice is safe and sensible to give to the driver. For example, telling a driver to accelerate harder when there is slower vehicle in front is counter-productive and would be unsafe.

The reader should note that the design is largely concentrated on cars with manual transmissions, since these represent the vast majority of cars sold in Europe. However, the system we describe could be adapted for cars with automatic transmissions without too much difficulty.

As well as measured variables, the proposed system requires various parameters to be set. These can be separated into two classes. The first class is vehicle and engine related. They take into account that different types of car are not identical. Important parameters of this type are the fuel consumption map of engine, gear ratios, vehicle weight, rolling resistance and air resistance.

The fuel consumption map is the key to the whole system. It is a three-dimensional plot of specific fuel consumption versus engine rotational velocity versus mean effective pressure (See Figure 1). Note that specific fuel consumption is defined as the ratio of useful power produced to the rate of fuel consumption. The fuel consumption map is usually represented in two dimensions by plotting equal specific fuel consumption contours on a graph which has the other two variables as axes. The lowest point of this contour map represents optimum fuel consumption and is known as the 'sweet spot'. One of the basic aims of the advice system is to keep the operating point of the engine as close to the sweet spot as possible, particularly during acceleration.

The second class of parameter is used to tune the behaviour of the system. Typical examples of such parameters are speed limits, minimum 'driveability' characteristics acceptable to the average driver, how long advice should be displayed for.

Data processing module

The data processing module is based around a concept known as a normative model. A schematic of the basic architecture of the data processing module is shown in Figure 2. This model describes the optimal driver behaviour for a wide range of contexts known as states. Determination of the current state is a key preprocessing task performed by the State Determination process. Typical states that are identified are: cruising, idling, decelerating, accelerating, gear changing. State determination is necessary because optimal driver behaviour depends heavily on the context in which the vehicle is being driven. Rules and advice on optimal driver behaviour should therefore apply to this context.

Actual driver behaviour is then compared with the optimal behaviour using the normative model. The architecture of the normative model is a multi-layered construction. The lowest layer is known as the tactical level and is concerned only with the immediate past. The next level up is known as the strategic level and uses a longer history of recorded measurements to provide a temporal context. 


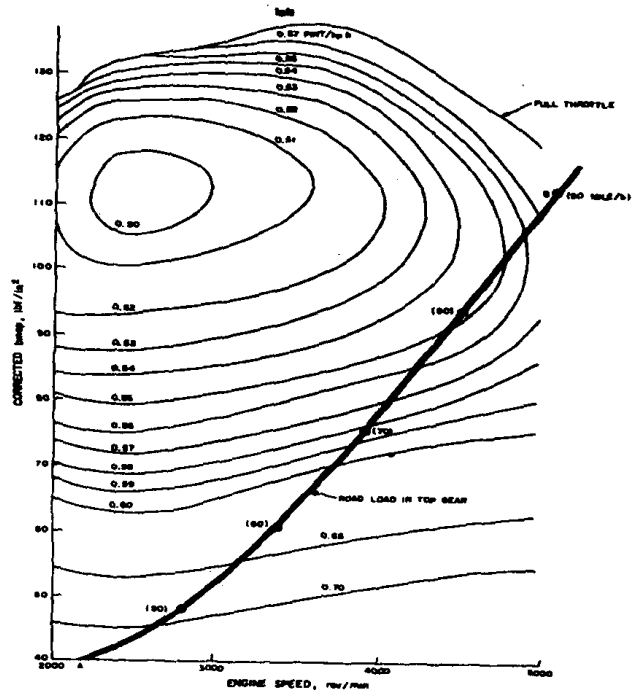

Figure 1 Typical fuel consumption map (2l-engine)

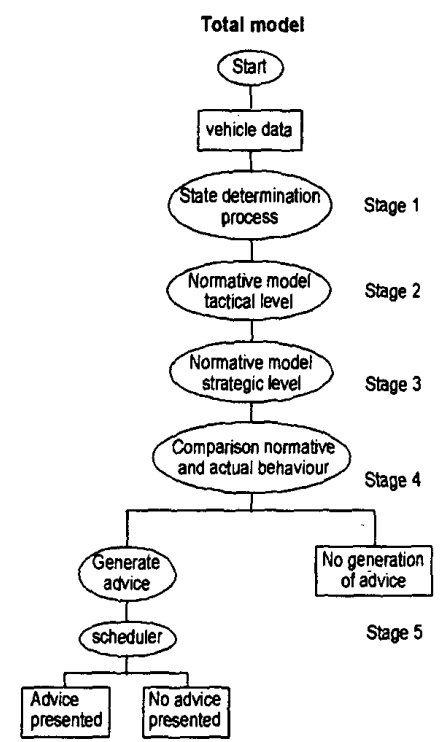

Figure 2 Architecture of the data processing module

The boundary between the immediate past and further back in time (which is dealt with by the strategic model) is defined as the last time a state change took place. A series of identical states is therefore grouped and defined as a manoeuvre. The unit of analysis for the tactical model is normally a single manoeuvre.

On the tactical level, for each type of manoeuvre, a normative model of optimal behaviour for minimum fuel consumption has been developed. Within these models, extensive use of the fuel consumption map is made.

The algorithms used are based on the following principles. More details can be found in [4].

1. The power profile needed to carry out a given manoeuvre (and associated fuel consumption profile for the engine) is calculated.

2. If no power is needed, the engine could be declutched or turned off, for example when approaching a stop. When stationary, it can often prove advantageous to switch off the engine.

3. A check is made whether the gear in use was the optimum gear. This is done by calculating the current fuel consumption and comparing what it would have been if a different gear had been used.

4. For manoeuvres, which involve acceleration or deceleration, it is examined whether better fuel consumption could have been achieved by changing the rate of acceleration/deceleration.

5. Excessive speed is discouraged, because of increased air resistance at higher speeds.

When determining the optimal behaviour, choice of accelerator pedal trajectory and appropriate gears through time (items 3 and 4 in the above list) become important factors, particularly during acceleration. In other words, to achieve optimum fuel consumption, it is not enough to solve the problem of minimising fuel consumption instantaneously; it has a temporal dimension.

The strategic level consist of a set of rules which have been derived from the studies about optimum fuel consumption referred to in [3]: It concentrates on identifying particular predefined sequences of manoeuvres:

1. do not start a trip with idling the engine for more than $10 \mathrm{sec}$

2. do not idle the engine for more than $15 \mathrm{sec}$; turn it off

3. do not creep forward and idle for more than $20 \mathrm{sec}$ in total

4. emergency braking should not occur more than twice per hour

5. acceleration should not be closely followed by deceleration

6. deceleration should not be closely followed by acceleration (unless you are turning).

If the difference in behaviour is large, a non-optimal behaviour is diagnosed. This in turn leads to advice being generated which is proposed to be presented to the driver by means of a suitable human-machine interface. To avoid presentation of negative advice only, positive feedback will be provided to the driver if he or she has driven fuel-efficiently for more than 4 minutes. 
Whether or when the advice is presented is determined by the scheduler. This scheduler has two roles.

a) Timing of advice: To ensure that different pieces of tactical and strategic advice do not conflict within a short period of time, which could prove confusing to the driver. Further, the same advice is not presented to the driver twice within a 3-minute period.

b) Safety check: To check (as far as is possible) that a particular piece of advice is not dangerous within the current driving context. For example, advising a driver to accelerate harder when the current headway is too small or presenting advice during emergency braking.

If the safety check fails, the advice is not presented. If the timing of advice is not appropriate, the advice will be restored into the buffer of the scheduler and will be reconsidered after a 1-second period. After 1 minute, the advice will be removed from the buffer.

\section{Human machine interfaces}

Two human-machine interfaces (HMIs) have been designed, which differ in the length of the advice given. A distinction is made between advice and extended advice. For extended advice, more details are provided to the driver. For instance, if the advice is: "Shift earlier", the matching extended advice could be: "Shift earlier from $2^{\text {nd }}$ to $3^{\text {rd }}$ gear". An example of the extended advice interface is shown in Figure 3.

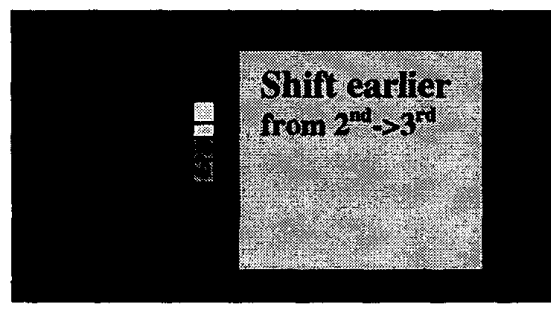

Figure 3 The human machine interface for extended advice

One of the key aspects of the driving simulator experiment, which will be described in the next section, was to determine which HMI is most effective.

\section{Driving Simulator Experiment}

The next phase of the research project was the evaluation of the new fuel-efficiency support tool with regard to its ability to reduce fuel consumption. A driving simulator experiment was carried out at the TNO Human Factors Research Institute in The Netherlands.

For the experiment the participants were divided into four groups. The two different human machine interfaces for the support tool (called the Advice group and the Extended Advice group) were assessed against two existing systems (called the Existing group) and a group which received no feedback (called the Control group).

The two existing systems are related to the miles-per-gallon meter. One showed every 3 minutes the average fuel consumption over the last 3-minute period, expressed in litres per 100 kilometres. The other also showed an indication of how fuel-efficient this figure was by means of green, orange and red LEDs.

In total, 88 subjects participated, equally divided over the four groups. All participants met the following criteria: male, between 25 and 45 years old, drove at least $10,000 \mathrm{~km}$ per year and no reading glasses. These criteria were used to identify a homogeneous set of participants.

Each participant drove 6 simulated journeys through urban, rural and highway environments. During the first run, all participants were asked to drive as they normally would. In the second run, participants were instructed to drive as fuel-efficiently as possible. However, they had to meet the constraint that travel time should remain constant, to avoid prompting people to start driving more slowly. During Runs 3-6, the participants (with exception of the Control group) received feedback from the support tool they were assigned to. For the Control group, the instruction during these runs was identical with Run 2. 
$\underline{\text { Results }}$

The results with regard to the fuel economy (in litres $/ 100 \mathrm{~km}$ ) measured over the total trip were subjected to a Kruskal-Wallis ANOVA by Ranks for each run. This ANOVA tests the hypothesis that the treatment medians do not differ significantly. This test was preferred above a standard ANOVA, since the assumption of equal standard deviations was violated during Run 3 (Levene's Test; $p=0.02$ ). The analysis revealed there exists no difference between the four groups during the first two runs. This means that the participants in the different groups act equally before the provision of feedback. In result, all differences found during Runs 3-6 are solely due to the presence of a feedback system.

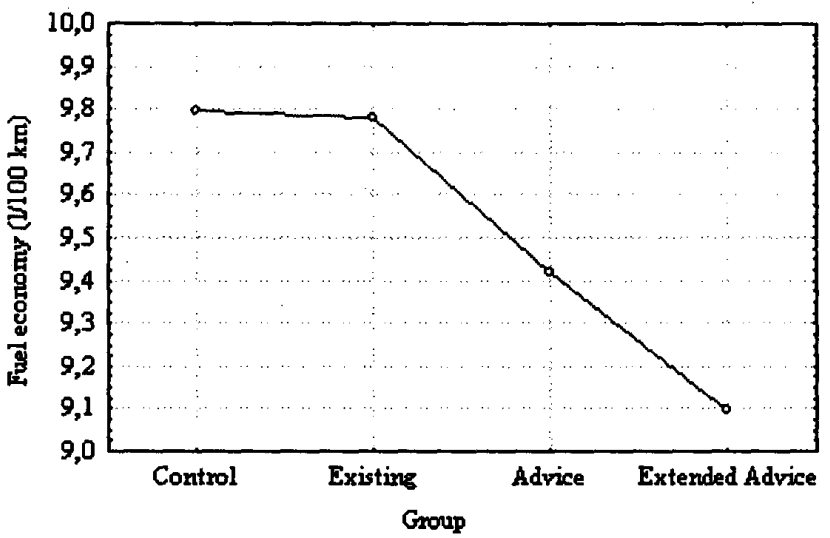

Figure $4 \quad$ Effect of Group on Fuel Economy $(\mathrm{l} / 100 \mathrm{~km})$ for the total trip

During Runs 3-6, significant differences between the groups were found $(p<0.005)$. A post-hoc multiple comparison (Tukey HSD) showed that only the group supported by the extended advice system drove significantly more fuel-efficiently than the Control group $(\mathrm{p}<0.01)$. This group also drove more fuel-efficiently than the group supported by the existing systems $(\mathrm{p}<0.01)$.

No significant difference was found between the Advice group and the Extended Advice group. This suggests that there is no effect of the length of the advice given. However, only the group with extended advice drove significantly more fuel-efficient than the Control group. Therefore it is inferred it is best to present the driver with extended advice.

If we express these results in a relative reduction of fuel consumption, the group supported by the extended advice system used up to $7 \%$ less fuel over Runs 3-6 than the Control group. The group using the existing devices was only able to reduce fuel consumption by $\sim 4 \%$. Compared with the fuel used during 'normal driving' (Run 1), drivers supported by the extended advice system are able to use $16 \%$ less fuel.

Even higher reductions were found under urban driving conditions. Figure 5 shows the effect of Group in the simulated urban environment over the 6 runs. In general, we can conclude that drivers are quite able to reduce fuel consumption by themselves (Run $1 \rightarrow$ Run 2). However, with support of an advice system, unlike using existing systems, drivers are able to reduce fuel consumption even further. In the urban environment, an additional reduction of $14 \%$ is found. Compared with 'normal driving' this yields a fuel reduction of $23 \%$.

Within the rural environment, results similar to the urban case were found. However, in this environment smaller reductions could be obtained. The Extended Advice group was able to obtain an additional reduction in fuel consumption of $\sim 4 \%$, compared with $\sim 2 \%$ for the Existing group.

On the highway section, no significant differences between the groups were found. The small impact within the rural and highway road categories could by explained by the fewer variables that drivers can change in their behaviour to reduce fuel consumption in these environments. Gear choice at high speeds is restricted to the highest gear. The driver can only influence his accelerator pedal position and by it speed. However, speed choice is restricted to the maximum legal speed limit or the speed of surrounding traffic. At low traffic volumes, no large differences in fuel economy are likely to be found. However, at higher traffic volumes, also within rural and highway road categories more variables of driver behaviour can and need to be changed. 


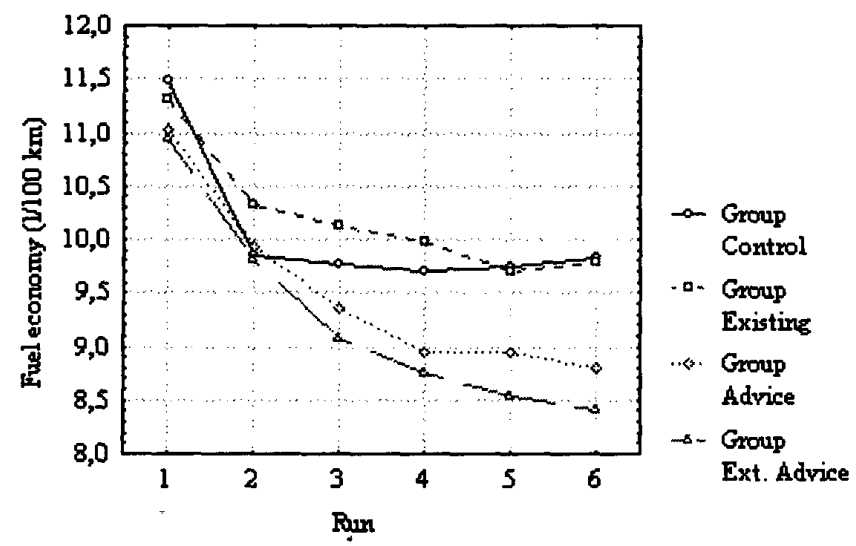

Figure 5 Fuel Economy $(\mathrm{l} / 100 \mathrm{~km})$ as a function of Group and Run in the urban environment

\section{Conclusions}

This research project has demonstrated that a fuel-efficiency support tool does help to reduce fuel consumption. However, there are two essential conditions: the system must provide the driver with detailed advice on how to drive fuel-efficiently and it must take into account the present context of the vehicle.

A new-generation fuel-efficiency support tool which satisfies these conditions has been designed. The support tool is based on a normative model, which identifies the present context of the vehicle and calculates the optimal behaviour within this context. If actual behaviour deviates from this optimal behaviour, the support tool presents advice to the driver on how to change his or her behaviour.

Through driving simulator experiment, the fuel reducing abilities of this new system have been tested against a control group and an existing device. The experiment revealed that with the new fuel-efficiency support tool, drivers are able to drive significantly more fuel-efficiently than without support or by using the existing devices. With the new tool, they gained, over the combined urban and non-urban cycle, a fuel reduction of $16 \%$ compared with 'normal driving'. Compared with driving fuel-efficiently without support, this means an additional reduction of $7 \%$. In the urban environment, reductions up to $22.5 \%$ were achieved.

These promising results will be verified on the road in a field trial by means of an instrumented vehicle or fleet study. The field trial will enable us to evaluate the impact of the system in and on real traffic conditions on a microscopic level. The field trial will also be utilised to assess the effects of the system on vehicle emissions.

\section{Acknowledgements}

The authors are grateful to the TNO Human Factors Research Institute at Soesterberg, The Netherlands, for the use of the driving simulator and in particular to Jeroen Hogema, Wytze Hoekstra and Richard van der Horst for the assistance, advice and generous giving of their time during the preparations and running of the experiment. The driving simulator experiment was sponsored by both KK-Stiftelsen, Sweden and the University of Twente.

\section{References}

[1] J: Decicco and M. Ross. "Recent advances in automotive technology and the cost-effectiveness of fuel economy improvement". Transportation Research Part D, Vol. 1, No. 2, 1996, p.79-96.

[2] M.H.L. Waters and I.B. Laker. Research on fuel conservation for cars. Report no. 921. Crowthorne, England, 1980.

[3] M.C. van der Voort. Generating a new fuel efficiency support tool-Motivation, state-of-the-art and salient features. CTS Working Paper 1997:9: Borlänge, Sweden, 1997.

[4] M.C. van der Voort \& M.S. Dougherty. A new generation fuel efficiency support tool. Paper presented at the TRB A1F03/A1F06 Joint Summer Meeting, Ann Arbor, Michigan. 2-4 August 1998. 